\title{
Ratchet universality in the bidirectional escape from a symmetric potential well
}

\author{
R. Chacón $\odot,{ }^{1,2}$ P. J. Martínez, ${ }^{3}$ J. M. Marcos $\odot,{ }^{2,4}$ F. J. Aranda, ${ }^{5}$ and J. A. Martínez ${ }^{6}$ \\ ${ }^{1}$ Departamento de Física Aplicada, Escuela de Ingenierías Industriales, Universidad de Extremadura, \\ Apartado Postal 382, E-06006 Badajoz, Spain \\ ${ }^{2}$ Instituto de Computación Científica Avanzada (ICCAEx), Universidad de Extremadura, E-06006 Badajoz, Spain \\ ${ }^{3}$ Departamento de Física Aplicada, E.I.N.A., Universidad de Zaragoza, E-50018 Zaragoza, Spain \\ and Instituto de Nanociencia y Materiales de Aragón (INMA), CSIC-Universidad de Zaragoza, E-50009 Zaragoza, Spain \\ ${ }^{4}$ Departamento de Física, Facultad de Ciencias, Universidad de Extremadura, E-06006 Badajoz, Spain \\ ${ }^{5}$ Departamento de Ingeniería Eléctrica, Electrónica y Automática, Facultad de Ciencias, \\ Universidad de Extremadura, E-06006 Badajoz, Spain \\ ${ }^{6}$ Departamento de Ingeniería Eléctrica, Electrónica y Automática, Escuela de Ingenieros Industriales, \\ Universidad de Castilla-La Mancha, E-02071 Albacete, Spain
}

(Received 16 December 2020; accepted 19 January 2021; published 4 February 2021)

\begin{abstract}
The present work discusses symmetry-breaking-induced bidirectional escape from a symmetric metastable potential well by the application of zero-average periodic forces in the presence of dissipation. We characterized the interplay between heteroclinic instabilities leading to chaotic escape and breaking of a generalized parity symmetry leading to directed ratchet escape to an attractor either at $\infty$ or at $-\infty$. Optimal enhancement of directed ratchet escape is found to occur when the wave form of the zero-average periodic force acting on the damped driven oscillator matches as closely as possible to a universal wave form, as predicted by the theory of ratchet universality. Specifically, the optimal approximation to the universal force triggers the almost complete destruction of the nonescaping basin for driving amplitudes which are systematically lower than those corresponding to a symmetric periodic force having the same period. We expect that this work could be potentially useful in the control of elementary dynamic processes characterized by multidirectional escape from a potential well, such as forced chaotic scattering and laser-induced dissociation of molecular systems, among others.
\end{abstract}

DOI: 10.1103/PhysRevE.103.022203

\section{INTRODUCTION}

Obtaining full control of the escape from a potential well is a problem of general interest in science, with broad technological implications in which the required energy to overcome the potential barrier can be supplied by both periodic and nonperiodic forces. Depending upon the force's features, escape can thus occur via the passage of the system over the potential barrier which separates the local potential minimum from one or several neighboring attracting domains. The energy required to surmount the potential barrier can be provided by different mechanisms, including the cases of noise-assisted and chaotic escapes. Diverse examples are known in distinct fields of chemical physics [1,2], electrical transport [3], astronomy and astrophysics [4-6], hydrodynamics [7-9], and quantum physics [10,11], among many others, in which escape phenomena can often be well described by a low-dimensional system of differential equations. Thus, a deterministic case that has been extensively studied in both dissipative and Hamiltonian systems is that where noise-free one-way escape is induced by an escape-inducing periodic force added to the low-dimensional model system, so that, before escape, chaotic transients of unpredictable duration (owing to the fractal character of the basin boundary) are usually observed for orbits starting from chaotic generic phase space regions (such as those surrounding separatrices). In this scenario, the effectiveness of secondary escape-taming periodic forces in suppressing one-way chaotic escape has been theoretically demonstrated for the case of the main resonance (between the two forces involved) in the context of dissipative systems capable of being studied by Melnikov analysis (MA) techniques [12,13], while its experimental effectiveness has also been demonstrated [14]. Moreover, the suppression of bidirectional chaotic escape from a symmetric quartic potential by secondary escape-taming forces has been demonstrated in the context of a damped-driven one-well Duffing oscillator [15]. In the last decades, the interest in the escape of chains of interacting oscillators out of metastable states [16-19] has grown in diverse scientific areas, although most of these studies have focused on the Hamiltonian limiting case.

The case of bidirectional escape from a symmetric potential well appears in diverse physical contexts, including solid-state turbulence in anisotropic solids [20], oscillating straight dislocation segments [21], and the boat capsize problem $[22,23]$, and provides a natural scenario to explore the control of ratchet escape (i.e., directed escape from a symmetric potential well by symmetry breaking of zero-mean forces). In this regard, the theory of ratchet universality [24-26] predicts that there exists a universal force wave form which 
optimally enhances directed transport by symmetry breaking. For deterministic ratchets, the effectiveness of the theory of ratchet universality has been demonstrated in diverse physical contexts in which the driving forces are chosen to be biharmonic, such as in the cases of cold atoms in optical lattices [27,28], topological solitons [29], Bose-Einstein condensates exposed to a sawtoothlike optical lattice potential [30], matter-wave solitons [31], and one-dimensional granular chains [32]. Also, the interplay between thermal noise and symmetry breaking in the directed ratchet transport (DRT) of a Brownian particle moving on a periodic substrate subjected to a homogeneous temporal biharmonic force [33-35] as well as the cases of a driven Brownian particle subjected to a vibrating periodic potential [26], a driven Brownian particle in the presence of non-Gaussian noise [36], and coupled Brownian motors with stochastic interactions in the crowded environment [37] have been explained quantitatively in coherence with the degree-of-symmetry-breaking (DSB) mechanism, as predicted by the theory of ratchet universality $[24,25]$.

In this present paper, we show that optimum enhancement of ratchet escape is achieved when maximal effective (i.e., critical) symmetry breaking occurs, i.e., when the wave form of the zero-average periodic force acting on the system matches as closely as possible to the exact universal wave form $[24,25]$. For the sake of clarity, we consider a simple paradigmatic model to discuss the bidirectional ratchet escape scenario: a damped-driven one-well Duffing oscillator described by the equation

$$
\ddot{x}+x-4 x^{3}=-\delta \dot{x}+\gamma F(t),
$$

where all the variables and parameters are dimensionless [20] $(\delta, \gamma>0)$, while $F(t)$ is a zero-average $T$-periodic external force. When the external force presents the shift symmetry, i.e., $F(t+T / 2)=-F(t)$, as in the case of a harmonic force for example, the damped-driven oscillator presents the generalized parity symmetry

$$
\mathbf{S}: x \rightarrow-x, t \rightarrow t+T / 2,
$$

i.e., if $[x(t), \dot{x}(t)]$ is a solution of Eq. (1), then so is $[-x(t+T / 2),-\dot{x}(t+T / 2)]$. This means that nonsymmetric stationary solutions always occur in pairs, including those escaping to the attractors at $\pm \infty$. Here, we deliberately choose an external force breaking such a generalized parity symmetry to investigate the directed ratchet escape (DRE) scenario:

$$
F(t)=F_{\text {ellip }}(t) \equiv \operatorname{sn}(\Omega t ; m) \operatorname{cn}(\Omega t ; m),
$$

where $\operatorname{cn}(\cdot ; m)$ and $\operatorname{sn}(\cdot ; m)$ are Jacobian elliptic functions [37] of parameter $m$, and $\Omega \equiv 2 K(m) / T$, with $K(m)$ being the complete elliptic integral of the first kind [38]. Fixing $T$, the wave form of $F_{\text {ellip }}(t) \equiv F_{\text {ellip }}(t ; T, m)$ changes as the shape parameter $m$ varies from 0 to 1 (see Fig. 1). Physically, the motivation for this choice is that $F_{\text {ellip }}(t ; T, m=$ $0)=\sin (2 \pi t / T) / 2$ and that $F_{\text {ellip }}(t ; T, m=1)$ vanishes; i.e., in these two limits DRE is not possible, while it is expected for $0<m<1$. Thus, one may expect that the strength of DRE to exhibit a maximum at a certain critical value $m=m_{c}$ as the shape parameter $m$ is varied, the remaining parameters being held constant. The DSB mechanism implies that such a

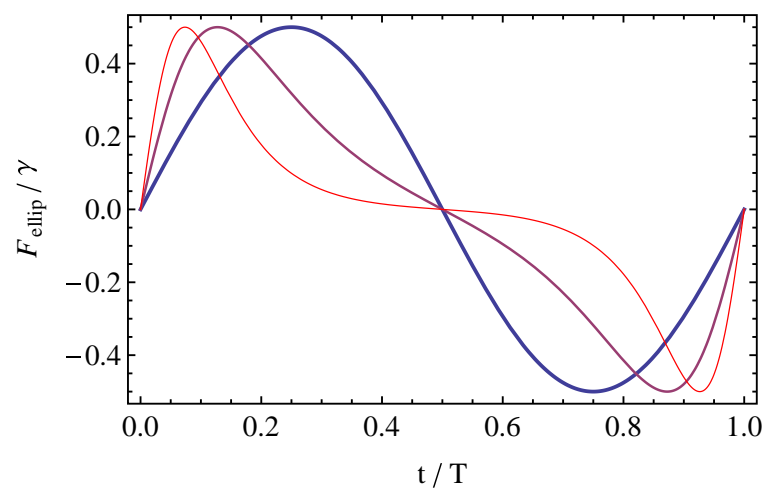

FIG. 1. Elliptic force $F_{\text {ellip }}(t)$ [cf. Eq. (3)] vs $t$ for three values of the shape parameter: $m=0,0.984$, and 0.9999 (solid curves of respectively decreasing thickness). The quantities plotted are dimensionless.

value $m_{c}$ corresponds to a particular force wave form which optimally enhances the ratchet effect. Furthermore, ratchet universality requires that such an optimal wave form should be closely related to that deduced for the case of a biharmonic force, in the sense of its Fourier series. Indeed, by using the Fourier series

$$
F_{\text {ellip }}(t)=\sum_{n=1}^{\infty} \frac{n \pi^{2} \operatorname{sech}\left[\frac{n \pi K(1-m)}{K(m)}\right] \sin (2 n \pi t / T)}{m K^{2}(m)},
$$

one could expect the critical value $m_{c}$ to be near $m=0.984$ since the optimal values for the biharmonic approximation of the elliptic function are recovered at $m=0.984$ (see Ref. [24] for additional details).

The rest of the paper is organized as follows. In the next section we obtain analytical estimates of the regions of the parameter space where chaotic escape events prompted by heteroclinic bifurcations can occur by using MA. The analysis of the interplay between such heteroclinic instabilities leading to chaotic escape and the breaking of the generalized parity symmetry leading to DRE to an attractor either at $\infty$ or at $-\infty$ is provided in Sec. III. Finally, Sec. IV is devoted to a discussion of the major findings and of some open problems.

\section{CHAOTIC ESCAPE THRESHOLD}

We assume that the complete system (1) satisfies the MA requirements, i.e., the dissipation and excitation terms are small-amplitude perturbations $(0<\delta, \gamma \ll 1)$ of the underlying conservative Duffing oscillator $\ddot{x}+x-4 x^{3}=0$ (see Refs. [39,40] for general background). It should be emphasized that the criterion for a homoclinic (or heteroclinic) tangency-accurately predicted by MA in diverse systems [7,15,41] - is coincident with the change from a smooth to an irregular, fractal-looking, basin boundary [42]. It is worth noting that these results connect MA predictions with those concerning the erosion of the basin boundary in phase space. 
Straightforward application of MA to Eqs. (1) and (4) yields the Melnikov function corresponding to the elliptic force:

$$
\begin{gathered}
M_{\text {ellip }}^{ \pm}\left(t_{0}\right)=-D \pm \sqrt{2} \gamma \sum_{n=1}^{\infty} a_{n}(m) b_{n}(T) \sin \left(\frac{2 n \pi t_{0}}{T}\right) \\
D \equiv \frac{\sqrt{2} \delta}{6} \\
a_{n}(m) \equiv \frac{n \pi^{2}}{m K^{2}(m)} \operatorname{sech}\left[\frac{n \pi K(1-m)}{K(m)}\right] \\
b_{n}(T) \equiv \frac{n \pi^{2}}{T} \operatorname{csch}\left[\frac{\sqrt{2} n \pi^{2}}{T}\right]
\end{gathered}
$$

where the positive (negative) sign refers to the top (bottom) heteroclinic orbit of the underlying conservative Duffing oscillator:

$$
x_{0}(t)= \pm \frac{1}{2} \tanh (\sqrt{2} t / 2), \dot{x}_{0}(t)= \pm \frac{\sqrt{2}}{4} \operatorname{sech}^{2}(\sqrt{2} t / 2) .
$$

If $M_{\text {ellip }}^{ \pm}\left(t_{0}\right)$ has a simple zero, i.e., there exists a value $t_{0}$ such that $M_{\text {ellip }}^{ \pm}\left(t_{0}\right)=0$ and $\partial M_{\text {ellip }}^{ \pm}\left(t_{0}\right) / \partial t_{0} \neq 0$, then a heteroclinic bifurcation occurs, signifying the possibility of bidirectional chaotic escape. From Eq. (5) one sees that a heteroclinic bifurcation is guaranteed if

$$
\frac{\delta}{\gamma}<U_{\text {ellip }}(m, T)
$$

where the chaotic threshold function is

$$
U_{\text {ellip }}(m, T) \equiv 6 \sum_{n=1}^{\infty} a_{n}(m) b_{n}(T)
$$

It is worth noting that condition (10) is the same for DRE to an attractor either at $+\infty$ or at $-\infty$. In other words, the chaotic threshold condition [Eq. (10)] does not provide information relating to the effective scape direction for a given set of parameters and initial conditions. Clearly, this is because condition (10) is the same for the two heteroclinic orbits of the underlying conservative Duffing oscillator. From Eq. (11) one readily obtains $U_{\text {ellip }}(m, T \rightarrow 0)=U_{\text {ellip }}(m \rightarrow$ $1, T)=0$; i.e., in such limits chaotic escape is not expected (see Fig. 2).

Let us consider the chaotic threshold as a function of $m$, holding $T$ constant. Plots of $U_{\text {ellip }}(m, T=$ const) show that each curve presents a single maximum $m_{\max }=m_{\max }(T)$ such that $m_{\max }(T)$ increases as $T$ is increased whenever $T$ is larger than a certain value $T^{*}$, while $U_{\text {ellip }}(m, T=$ const) is a monotonically decreasing function of $m$ whenever $T<T^{*}$ [see Figs. 2(a) and 2(c)]. Therefore, if one considers fixing the parameters $\left(\delta, \gamma, T>T^{*}\right)$ for the system to lie at a periodic state (i.e., inside the well), then as $m$ is increased a window of chaotic escape will appear provided the initial periodic state is sufficiently near the chaotic regime. We now study the chaotic threshold as a function of $T$, holding $m$ constant. Plots of $U_{\text {ellip }}(m=$ const, $T)$ show that each curve asymptotically tends to a constant value which depends on $m$ :

$$
U_{\text {ellip }}(m=\text { const, } T \rightarrow \infty) \sim \frac{6}{\sqrt{2}} \sum_{n=1}^{\infty} a_{n}(m) \leqslant \pi
$$
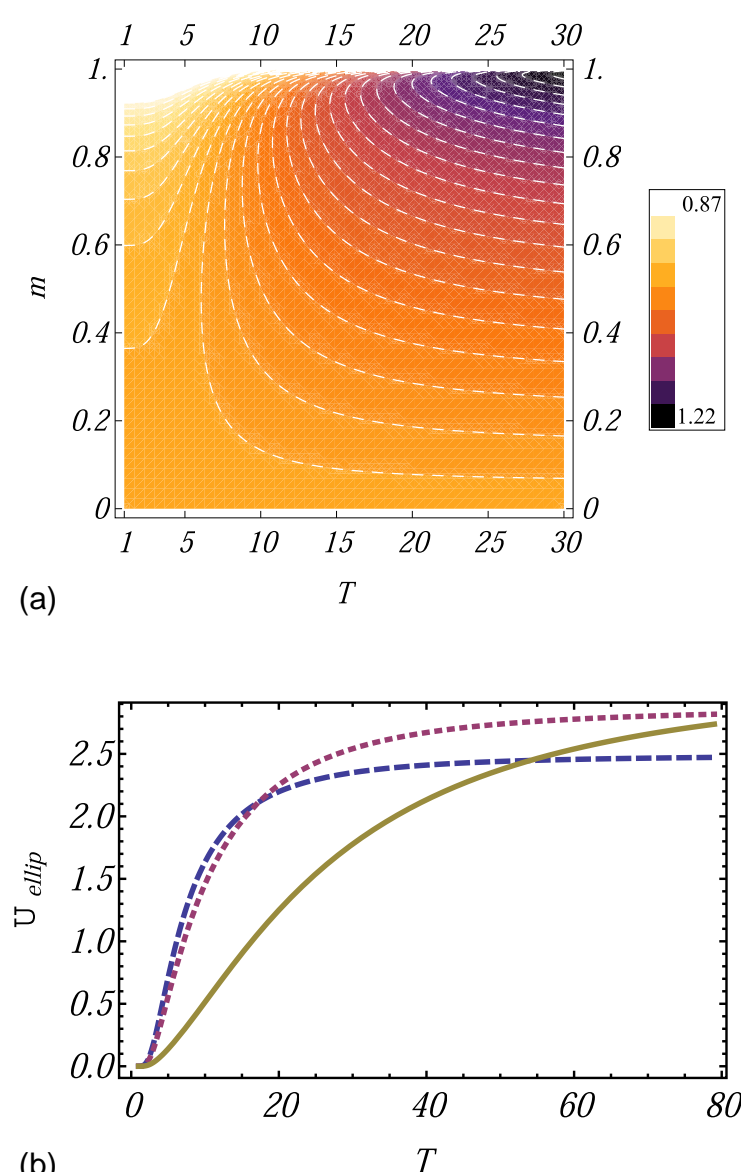

(b)

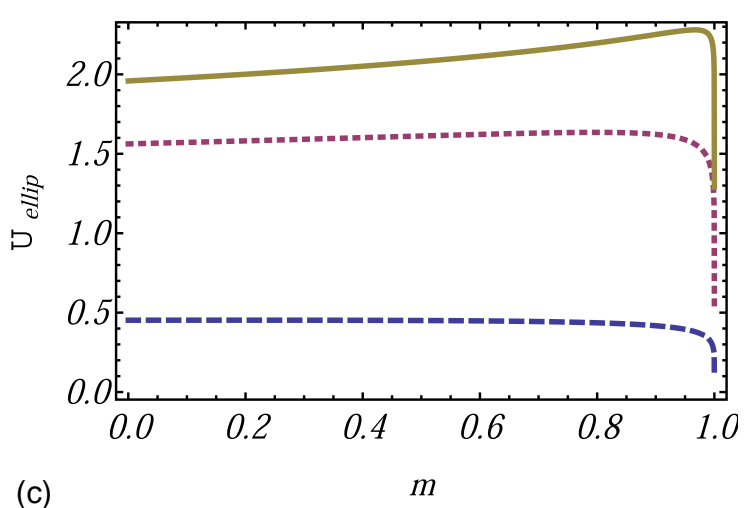

FIG. 2. (a) Contour plot of the chaotic threshold function associated with the elliptic force $U_{\text {ellip }} \equiv U_{\text {ellip }}(m, T)$ [cf. Eq. (11)] vs the shape parameter $m$ and the driving period $T$. (b) Chaotic threshold function $U_{\text {ellip }} \equiv U_{\text {ellip }}(m, T)$ [cf. Eq. (11)] vs the driving period $T$ for $m=0.8$ (dashed line), $m=0.99$ (dotted line), and $m=1-10^{-8}$ (solid line). (c) Chaotic threshold function $U_{\text {ellip }} \equiv U_{\text {ellip }}(m, T)$ [cf. Eq. (11)] vs the shape parameter $m$ for $T=4$ (dashed line), $T=10$ (dotted line), and $T=20$ (solid line). The quantities plotted are dimensionless.

see Fig. 2(b). This means that chaotic escape is facilitated (i.e., one needs smaller values of the amplitude) when the driving period is relatively large, holding $\delta$ and $m$ constant [cf. Eq. (10)]. 


\section{SYMMETRY-BREAKING-INDUCED ESCAPE}

We explore in this section the effectiveness of the force $F_{\text {ellip }}(t)$ [Eq. (3)] at controlling the strength of DRE in Eq. (1). It is worth recalling that the existence of a universal wave form for optimal enhancement of DRT is a direct consequence of the DSB mechanism: It is possible to consider a quantitative measure of the DSB on which the strength of directed transport by symmetry breaking must depend. This mechanism has led to the unveiling of a criticality scenario for DRT. Indeed, it has been shown that optimal enhancement of DRT is achieved when maximal effective (i.e., critical) symmetry breaking occurs, which is in turn a consequence of two reshaping-induced competing effects: the increase of the DSB and the decrease of the (normalized) maximal transmitted impulse over a halfperiod $\left(I[f] \equiv\left|\int_{T / 2} f(t) d t\right|\right.$; see Refs. [24,25] for additional details), thus implying the existence of a particular force wave form which optimally enhances DRT. The definitions of the DSB of the symmetries of a $T$-periodic zero-mean ac force $f(t)$ are included here for the sake of completeness:

$$
\begin{aligned}
D_{s}[f] & \equiv\left\langle\frac{-f(t+T / 2)}{f(t)}\right\rangle_{T} \equiv \frac{1}{T} \int_{0}^{T} \frac{-f(t+T / 2)}{f(t)} d t, \\
D_{+}[f] & \equiv\left\langle\frac{f(-t)}{f(t)}\right\rangle_{T} \equiv \frac{1}{T} \int_{0}^{T} \frac{f(-t)}{f(t)} d t, \\
D_{-}[f] & \equiv-D_{+}[f],
\end{aligned}
$$

where increasing deviation of $D_{s,+,-}[f]$ from 1 (unbroken shift and reversal symmetries, respectively) indicates an increase in the DSB (see Refs. [24,25] for additional details). In the case of the elliptic force $F_{\text {ellip }}(t)$, such reshaping-induced competing effects are clearly operating when $m$ varies between 0 and 1 , while the optimal enhancement of the ratchet effect is expected to occur at the critical value $m_{c} \simeq 0.984$, as already explained in Sec. I. Indeed, we found that the impulse transmitted by the elliptic force per unit of period over a half-period

$$
I \equiv \frac{1}{T} \int_{0}^{T / 2} F_{\mathrm{ellip}}(t) d t=[2 K(m)(1+\sqrt{1-m})]^{-1}
$$

is a monotonously decreasing function of the shape parameter, while the quantifier of the DSB associated with its shift symmetry is a monotonously increasing function of the shape parameter [cf. Eq. (13)]:

$$
D_{s}\left[F_{\text {ellip }}\right]=\frac{2 E(m)}{\sqrt{1-m} K(m)},
$$

where $E(m)$ is the complete elliptic integral of the second kind [38] (see Fig. 3).

For the bidirectional escape model (1), the initial conditions will determine, for a fixed set of its parameters, whether the system escapes to an attractor at $\pm \infty$ or settles into a bounded oscillation. Similarly to the case of noise-free one-way escape [7], there can exist a rapid and dramatic erosion of the safe basin (union of the basins of the bounded attractors) due to encroachment by the basins of the attractors at $\pm \infty$ (escaping basins). The basins of attraction were computed using a fourth-order Runge-Kutta algorithm with time steps in the range $\Delta t=0.005-0.01$. To numerically

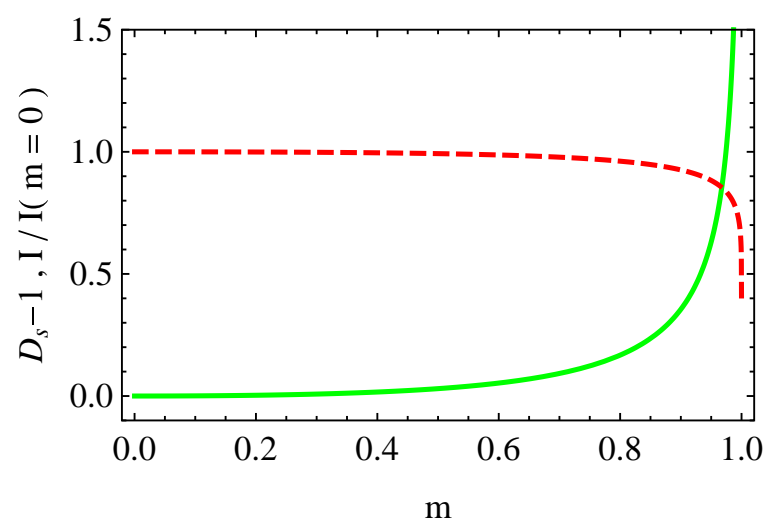

FIG. 3. Quantifier of the DSB associated with the shift symmetry $D_{s}$ [cf. Eq. (15); solid line] and impulse transmitted by the elliptic force per unit of period over a half-period [cf. Eq. (14); dashed line] vs the shape parameter $m$. The quantities plotted are dimensionless.

generate the basins of attraction, we considered a grid of (uniformly distributed) $401 \times 401$ starting points in the region of phase space $x(t=0) \in[-0.5,0.5]$ and $\dot{x}(t=0) \in$ $[-0.3535,0.3535]$, and we selected those initial conditions inside the region bounded by the separatrix formed by the two heteroclinic orbits [Eq. (9)]. From this selected set of initial conditions, each integration is continued until either $x(-x)$ exceeds 5 , at which point the system is deemed to have escaped to the attractor at $\infty(-\infty)$, or the maximum allowable number of cycles, here 20 , is reached. To provide a quantitative measure of the DRE strength, we calculated the escape probabilities $P_{ \pm}$associated with escape to the attractors at $\pm \infty$, respectively, and the total escape probability $P_{T}=P_{+}+P_{-}$versus the shape parameter $m$. The escape probability is $P_{ \pm} \equiv N_{ \pm} / N_{\text {sep }}$, where $N_{ \pm}$is the (corresponding) number of starting points from which the system is deemed to have escaped and $N_{\text {sep }}=106673$ is the number of starting points inside the separatrix according to the aforementioned criterion.

In the case of a shift-symmetric (harmonic) force $(m=0)$, we assume that the system presents a slight erosion of the nonescaping basin for a fixed set of parameters $(\delta, \gamma, T)$ satisfying the chaotic threshold condition [cf. Eq. (10)]. Notice that the escape probabilities $P_{+}(m=0)$ and $P_{-}(m=0)$ are expected to be different because of the temporal shift between the corresponding solutions escaping to $\infty$ and $-\infty$ [cf. Eq. (2)]. Figure 4 shows an illustrative example comparing the cases corresponding to six values of the shape parameter $m=0,0.8443,0.8665 \approx m_{\max }(T=2 \pi / 0.5268)$, $0.984 \approx m_{c}, 0.9895,0.998$ [cf. Figs. 4(a) to 4(f), respectively] for the parameters $\delta=0.2, \gamma=0.28$, and $T=2 \pi / 0.5268$. Our numerical experiments have typically shown that the escape probability $P_{+}$can present one or two maxima as the shape parameter $m$ is increased from 0 , the remaining parameters being held constant [see Fig. 5(a)]. The lower local maximum, $m_{\max }^{\text {chaos }}$, which can exist or not depending upon the particular value of $T$ (cf. Sec. II), is systematically associated with the corresponding maximum $m_{\max }=m_{\max }(T)$ of the chaotic threshold function [Eq. (11)]. Indeed, the wave forms associated with the values $m=0.8443 \approx m_{\max }^{\text {chaos }}$ and 

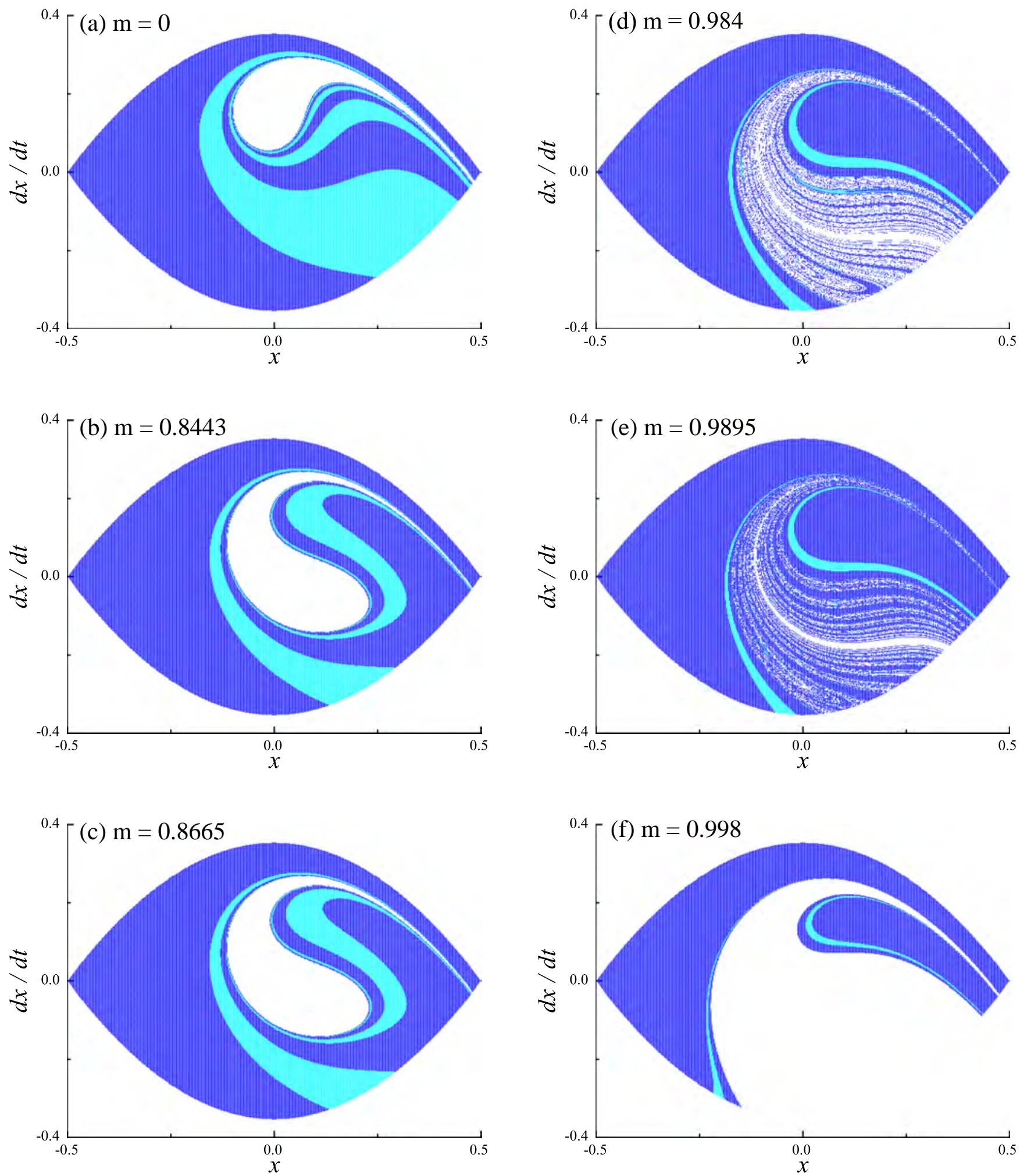

FIG. 4. Escaping and nonescaping basins of the one-well Duffing oscillator subjected to the elliptic force $F_{\text {ellip }}(t ; T, m)$ [cf. Eqs. (1) and (3)] for $\delta=0.2, \gamma=0.28, T=2 \pi / 0.5268$, and six values of the shape parameter: $m=0(\mathrm{a}), m=0.8443 \approx m_{\max }^{\text {num }}(\mathrm{b}), m=0.8665 \approx m_{\max }(T=$ $2 \pi / 0.5268$ ) (c), $m=0.984 \approx m_{c}(\mathrm{~d}), m=0.9895 \approx m_{\max }^{\text {ratchet }}(\mathrm{e})$, and $m=0.998$ (f). The color cyan (pale gray) represents the escaping basin towards the attractor at $-\infty$, the color blue (black) represents the escaping basin towards the attractor at $\infty$, and the blank regions represent the nonescaping basin. The quantities plotted are dimensionless. 

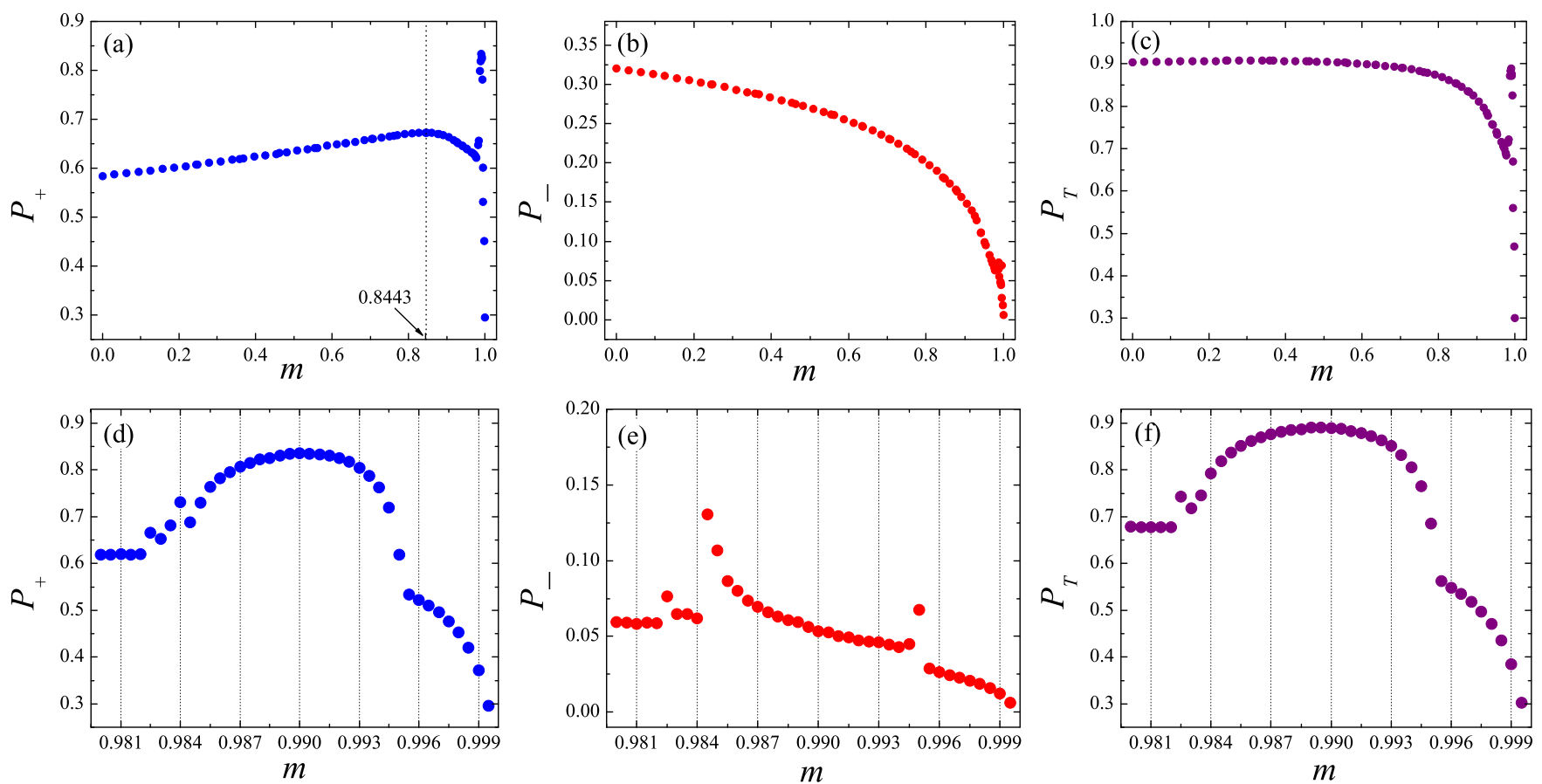

FIG. 5. Escape probabilities $P_{+}$(a), $P_{-}$(b), and $P_{T}$ (c) (see the text) vs the shape parameter $m$. Versions (d), (e), and (f) show enlargements of the versions (a), (b), and (c) over the range $0.98<m<1$, respectively. System parameters: $\delta=0.2, \gamma=0.28$, and $T=2 \pi / 0.5268$. The quantities plotted are dimensionless.

$0.8665 \approx m_{\max }(T=2 \pi / 0.5268)$ are hardly distinguishable, which is a consequence of the dependence of $K(m)$ on $m$ [38], and thereby the respective escaping and nonescaping basins are quite similar [compare Figs. 4(b) and 4(c)]. The fact that the escape probability $P_{-}$does not present a local maximum at $m_{\max }^{\text {chaos }}$ [cf. Fig. 5(b)] means that such a local maximum of $P_{+}$ is a result of the conjoint effects of heteroclinic instabilities and DRE. The absolute maximum of $P_{+}$is systematically associated with the critical value $m_{c}$ (cf. Sec. I), as is shown in Figs. 5(a) and 5(d). Similarly to the relative maximum $m_{\max }^{\text {chas }}$, one finds that the absolute maximum is not associated with a sharp peak of the escape probability, but rather it is nearly a plateau over a certain short range of the shape parameters which is very close to the value $m=0.984$. Again, note that the wave forms associated with the values $0.984 \approx m_{c}$ and $0.9895 \approx m_{\max }^{\text {ratchet }}$ (absolute maximum) are hardly distinguishable for the same aforementioned reason, the respective escaping and nonescaping basins being therefore quite similar [compare Figs. 4(d) and 4(e)]. Figures 6(a) and 6(b) show that the fractal-like fingers protruding into the nonescaping basin are similar for these two $m$ values. While the condition for the onset of heteroclinic instabilities [cf. Eqs. (10) and (11) with $\delta=0.2, \gamma=0.28, T=2 \pi / 0.5268$ ], and hence for the appearance of fractal basin boundaries, is satisfied over a wide range of shape parameters containing the window $m \in\left[0, m_{\max }^{\text {ratchet }}\right]$, one finds that the large-scale destruction of the nonescaping basin solely occurs around $m_{\max }^{\text {ratchet }}$. Remarkably, the escape probability $P_{-}$presents sharp local maxima at values of $m$ near the edges of the aforementioned plateau [see Fig. 5(e)], while it presents an overall decreasing behavior as a function of the shape parameter from $m=0$ because of the ratchet effect [see Fig. 5(b)]. Also, all the escape probabilities $P_{+}, P_{-}$, and $P_{T}$ present a decreasing behavior as $m \rightarrow 1$ (see Fig. 5) because the impulse transmitted by the elliptic force per unit of period over a half-period [Eq. (14)] is a monotonously decreasing function of the shape parameter and $F_{\text {ellip }}(t ; T, m=1)$ vanishes. It is worth mentioning that we found similar results for other sets of parameters, i.e., the enhancement of the dramatic erosion and stratification of the nonescaping basin is a genuine feature of the DRE scenario associated with the universal force wave form [compare Figs. 4(a) and 4(d)]. Furthermore, we found that the optimal ratcheting force $F_{\text {ellip }}\left(t ; T, m=m_{\max }^{\text {ratchet }}\right)$ triggers the almost complete destruction of the nonescaping basin for driving amplitudes which are systematically lower than those corresponding to the shift-symmetric (harmonic) force $F_{\text {ellip }}(t ; T, m=0)$ [compare Figs. 4(e), 7(b), and 7(c)].

\section{CONCLUSIONS}

In summary, we have investigated the effectiveness of zeroaverage periodic forces at yielding directed ratchet escape from a symmetric potential well by considering an asymmetric external periodic force. Optimal enhancement of directed ratchet escape is predicted to occur when the wave form of the zero-average periodic force acting on a damped driven oscillator matches as closely as possible to a biharmonic universal wave form, as predicted by the theory of ratchet universality. Our numerical experiments confirmed those findings, as well as revealed the interplay between heteroclinic instabilities leading to chaotic escape and breaking of a generalized parity symmetry leading to directed ratchet escape to an attractor either at $\infty$ or at $-\infty$. Specifically, the optimal approximation to the biharmonic universal force triggers the almost complete destruction of the nonescaping basin for driving amplitudes which are systematically lower than those corresponding to a symmetric periodic force having the same period. 
(a) $\mathrm{m}=0.984$

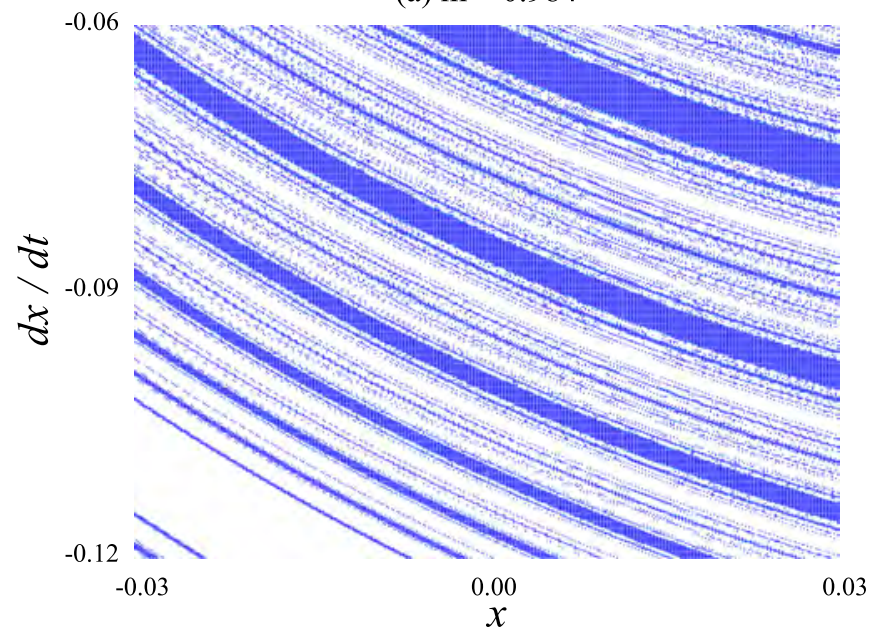

(b) $\mathrm{m}=0.9895$

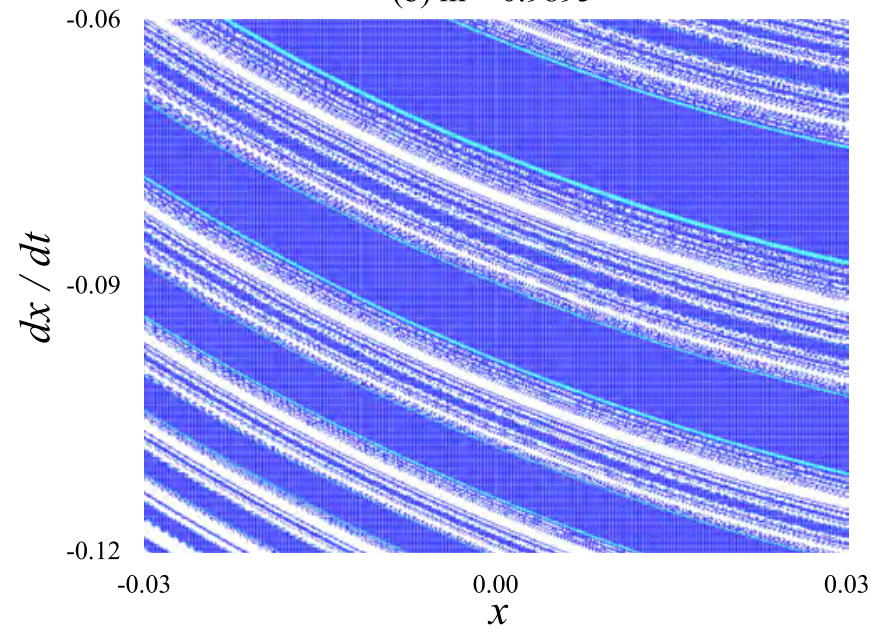

FIG. 6. Detail of the escaping and nonescaping basins of the onewell Duffing oscillator subjected to the elliptic force $F_{\text {ellip }}(t ; T, m)$ [cf. Eqs. (1) and (3)] corresponding to the windows $-0.03 \leqslant x \leqslant$ 0.03 and $-0.12 \leqslant \dot{x} \leqslant-0.06$ for $m=0.984 \approx m_{c}$ (a) and $m=$ 0.9895 (b) [cf. Figs. 4(d) and 4(e), respectively]. The color cyan (pale gray) represents the escaping basin towards the attractor at $-\infty$, the color blue (black) represents the escaping basin towards the attractor at $\infty$, and the blank regions represent the nonescaping basin. System parameters: $\delta=0.2, \gamma=0.28$, and $T=2 \pi / 0.5268$. The quantities plotted are dimensionless.

We should emphasize that the directed-ratchet-escape scenario we have discussed is general enough to be applied to many other dissipative nonautonomous systems. Specifically, such a scenario can be readily tested experimentally (for instance, in driven quantum Josephson circuits [11]) and can find application for improving the control of elementary dynamic processes characterized by multidirectional escape from a potential well, such as forced chaotic scattering [43] and transport phenomena in dissipative lattices as well as diverse atomic and molecular processes [44]. Additionally, a natural extension of this work would be to investigate the directed ratchet escape of a chain of coupled driven oscillators over the barriers of a metastable symmetric potential both in the presence and in the absence of dissipation (Hamiltonian
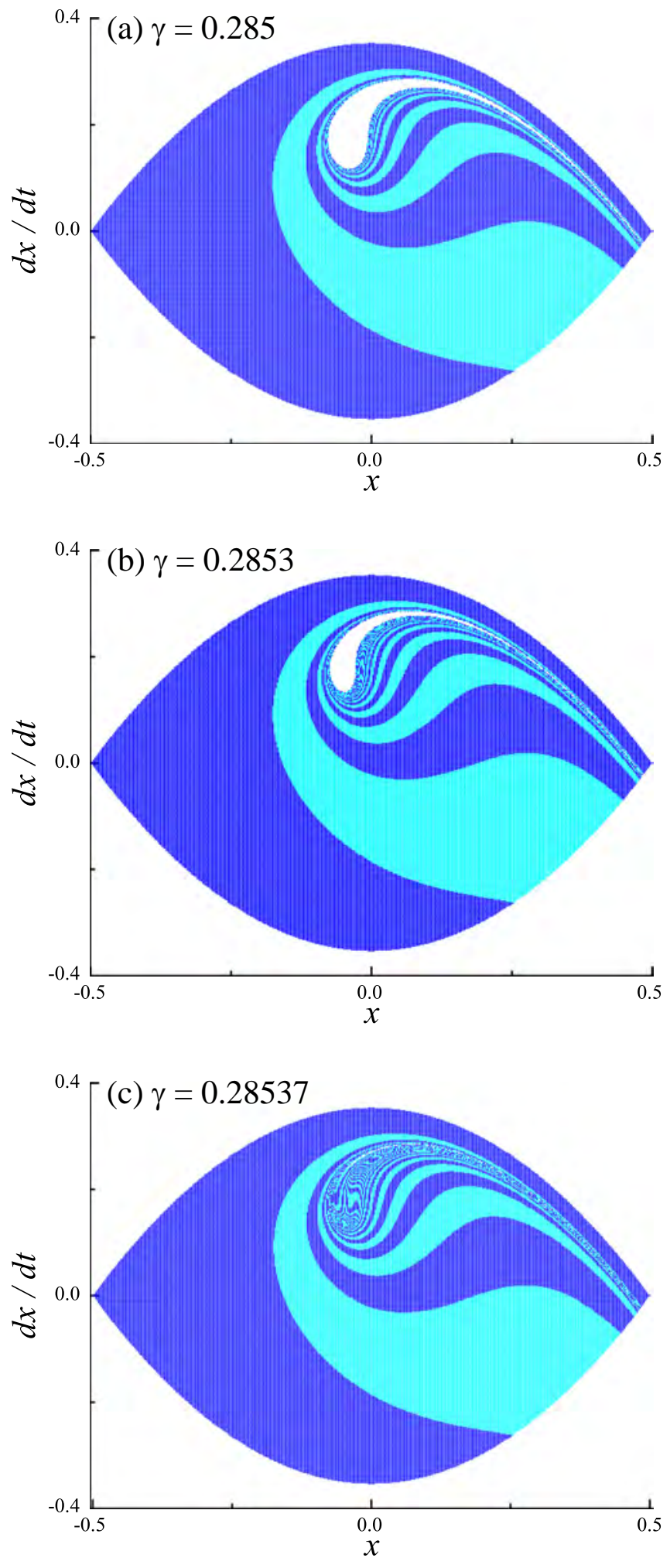

FIG. 7. Escaping and nonescaping basins of the one-well Duffing oscillator subjected to the shift-symmetric (harmonic) force $F_{\text {ellip }}(t ; T, m=0)$ [cf. Eqs. (1) and (3)] for $\delta=0.2, T=2 \pi / 0.5268$, and three values of the driving amplitude: $\gamma=0.285$ (a), $\gamma=0.2853$ (b), and $\gamma=0.28537$ (c). The color cyan (pale gray) represents the escaping basin towards the attractor at $-\infty$, the color blue (black) represents the escaping basin towards the attractor at $\infty$, and the blank regions represent the nonescaping basin. The quantities plotted are dimensionless. 
limiting case). We would like to investigate this issue in the near future.

\section{ACKNOWLEDGMENTS}

R.C. acknowledges financial support from the Junta de Extremadura (JEx, Spain) through Project No. GR18081 cofinanced by FEDER funds. P.J.M. acknowledges financial support from the Ministerio de Economía y Competitividad (MINECO, Spain) through Project No. FIS2017-87519 cofinanced by FEDER funds and from the Gobierno de Aragón (DGA, Spain) through Grant No. E36_17R to the FENOL group.
[1] R. Ramaswamy, P. Siders, and R. A. Marcus, A simple classical model of infrared multiphoton dissociation, J. Chem. Phys. 74, 4418 (1981).

[2] M. E. Goggin and P. W. Milonni, Driven Morse oscillator: Classical chaos, quantum theory, and photodissociation, Phys. Rev. A 37, 796 (1988).

[3] I. Vernik, S. Keil, N. Thyssen, T. Doderer, A. V. Ustinov, H. Kohlstedt, and R. P. Huebener, Fluxon pinning in annular Josephson junctions by an external magnetic field, J. Appl. Phys. 81, 1335 (1997).

[4] G. Contopoulos, H. Handrup, and D. Kaufmann, Fractal properties of escape from a two-dimensional potential, Phys. D (Amsterdam, Neth.) D64, 310 (1993).

[5] S. Astakhov, A. Burbanks, S. Wiggins, and D. Farrelly, Chaosassisted capture of irregular moons, Nature (London) 423, 264 (2003).

[6] S. Astakhov and D. Farrelly, Capture and escape in the elliptic restricted three-body problem, Mon. Not. R. Astron. Soc. 354, 971 (2004).

[7] J. M. T. Thompson, Chaotic phenomena triggering the escape from a potential well, Proc. R. Soc. A 421, 195 (1989).

[8] J. M. T. Thompson, S. R. Bishop, and L. M. Leung, Fractal basins and chaotic bifurcations prior to escape from a potential well, Phys. Lett. A 121, 116 (1987).

[9] J. M. T. Thompson and M. S. Soliman, Fractal control boundaries of driven oscillators and their relevance to safe engineering design, Proc. R. Soc. Lond. A 428, 1 (1990).

[10] R. Chacón and J. I. Cirac, Chaotic and regular behavior of a trapped ion interacting with a laser field, Phys. Rev. A 51, 4900 (1995).

[11] R. Lescanne, L. Verney, Q. Ficheux, M. H. Devoret, B. Huard, M. Mirrahimi, and Z. Leghtas, Escape of a Driven Quantum Josephson Circuit into Unconfined States, Phys. Rev. Appl. 11, 014030 (2019).

[12] R. Chacón, F. Balibrea, and M. A. López, Inhibition of chaotic escape from a potential well using small parametric modulations, J. Math. Phys. 37, 5518 (1996).

[13] R. Chacón and J. A. Martínez, Controlling escape from a potential well by reshaping periodic secondary excitations, Phys. Rev. E 83, 016201 (2011).

[14] J. M. Seoane, S. Zambrano, S. Euzzor, R. Meucci, F. T. Arecchi, and M. A. Sanjuán, Avoiding escapes in open dynamical systems using phase control, Phys. Rev. E 78, 016205 (2008).

[15] R. Chacón, F. Sánchez-Bajo, and J. A. Martínez, Robustness in the suppression of bidirectional chaotic escape from a potential well by weak parametric excitations, Phys. Lett. A 303, 190 (2002).

[16] P. J. Park and W. Sung, Polymer release out of a spherical vesicle through a pore, Phys. Rev. E 57, 730 (1998).
[17] S. Martens, D. Henning, S. Fugmann, and L. SchimanskyGeier, Resonancelike phenomena in the mobility of a chain of nonlinear coupled oscillators in a two-dimensional periodic potential, Phys. Rev. E 78, 041121 (2008).

[18] K. Manski and D. Henning, Escape dynamics of coupled particles in nonlinear, disordered lattices, Phys. Rev. E 80, 051109 (2009).

[19] T. Gross, D. Henning, and L. Schimansky-Geier, Modulational instability and resonant wave modes act on the metastability of oscillator chains, Phys. Rev. E 90, 032919 (2014).

[20] B. A. Huberman and J. P. Crutchfield, Chaotic States of Anharmonic Systems in Periodic Fields, Phys. Rev. Lett. 43, 1743 (1979).

[21] C. Herring and B. A. Huberman, Dislocation motion and solidstate turbulence, Appl. Phys. Lett. 36, 975 (1980).

[22] L. N. Virgin, Approximate criterion for capsize based on deterministic dynamics, Dyn. Stab. Syst. 4, 55 (1989).

[23] A. Maki, L. N. Virgin, N. Umeda, T. Ueta, Y. Miino, M. Sakai, and $\mathrm{H}$. Kawakami, On the loss of stability and its relevance to ship capsize, J. Mar. Sci. Technol. 24, 846 (2019).

[24] R. Chacón, Optimal control of ratchets without spatial asymmetry, J. Phys. A 40, F413 (2007).

[25] R. Chacón, Criticality-induced universality in ratchets, J. Phys. A 43, 322001 (2010).

[26] R. Chacón and P. J. Martínez, Exact universal excitation waveform for optimal enhancement of directed ratchet transport [Int. J. Bifurcation Chaos (to be published)].

[27] M. Schiavoni, L. Sánchez-Palencia, F. Renzoni, and G. Grynberg, Phase Control of Directed Diffusion in a Symmetric Optical Lattice, Phys. Rev. Lett. 90, 094101 (2003).

[28] R. Chacón, Comment on "Phase Control of Directed Diffusion in a Symmetric Optical Lattice," arXiv:1802.02826.

[29] P. J. Martínez and R. Chacón, Disorder Induced Control of Discrete Soliton Ratchets, Phys. Rev. Lett. 100, 144101 (2008).

[30] T. Salger, S. Kling, T. Hecking, C. Geckeler, L. MoralesMolina, and M. Weitz, Directed transport of atoms in a Hamiltonian quantum ratchet, Science 326, 1241 (2009).

[31] M. Rietmann, R. Carretero-González, and R. Chacón, Controlling directed transport of matter-wave solitons using the ratchet effect, Phys. Rev. A 83, 053617 (2011).

[32] V. Berardi, J. Lydon, P. G. Kevrekidis, C. Daraio, and R. Carretero-González, Directed ratchet transport in granular chains, Phys. Rev. E 88, 052202 (2013).

[33] P. J. Martínez and R. Chacón, Ratchet universality in the presence of thermal noise, Phys. Rev. E 87, 062114 (2013).

[34] P. J. Martínez and R. Chacón, Erratum: Ratchet universality in the presence of thermal noise [Phys. Rev. E 87, 062114], Phys. Rev. E 88, 019902(E) (2013). 
[35] P. J. Martínez and R. Chacón, Reply to "Comment on 'Ratchet universality in the presence of thermal noise',' Phys. Rev. E 88, 066102 (2013).

[36] J. Xu and X. Luo, Ratchet effects of a Brownian particle with non-Gaussian noise driven by a biharmonic force, Mod. Phys. Lett. B 33, 1950230 (2019).

[37] L. Lin, L. Yu, W. Lv, and H. Wang, Ratchet motion and current reversal of Brownian motors coupled by birth-death interactions in the crowded environment, Chin. J. Phys 68, 808 (2020).

[38] P. F. Byrd and M. D. Friedman, Handbook of Elliptic Integrals for Engineers and Scientists (Springer-Verlag, Berlin, 1971).

[39] V. K. Melnikov, Tr. Mosk. Ova. 12, 3 (1963) [Trans. Moscow Math. Soc. A 12, 1 (1963)].
[40] J. Guckenheimer and P. J. Holmes, Nonlinear Oscillations, Dynamical Systems, and Bifurcations of Vector Fields (SpringerVerlag, New York, 1983)

[41] F. C. Moon and G. X. Li, Fractal Basin Boundaries and Homoclinic Orbits for Periodic Motion in a Two-Well Potential, Phys. Rev. Lett. 55, 1439 (1985).

[42] S. W. McDonald, C. Grebogi, E. Ott, and J. A. York, Phys. D (Amsterdam, Neth.) 17, 125 (1985).

[43] A. R. Nieto, J. M. Seoane, J. E. Alvarellos, and M. A. F. Sanjuán, Resonant behavior and unpredictability in forced chaotic scattering, Phys. Rev. E 98, 062206 (2018).

[44] Y. Gu and J.-M. Yuan, Classical dynamics and resonance structures in laser-induced dissociation of a Morse oscillator, Phys. Rev. A 36, 3788 (1987). 